\title{
LARGE ABELIAN SUBGROUPS OF SOME INFINITE GROUPS, II
}

\author{
V. FABER
}

1. Introduction. This paper extends the work of [2], [3], and [4]. The main theorems are:

THEOREM 1. If $G$ is an infinite group of cardinality $m^{+}$having a (strictly increasing) normal series $\left(G_{\alpha}\right)$ with $\left|G_{\alpha}\right| \leqq m$ for all $\alpha$, then every maximal abelian subgroup A of $G$ satisfies $m^{|A|} \geqq|G|$.

THEOREM 6. Every infinite $F C N^{*}$ group has an abelian subgroup $A$ such that $\exp |A| \geqq|G|$.

THEOREM 9. Every maximal abelian subgroup A of an uncountable ZA group $G$ satisfies $\exp |A| \geqq|G|$.

THEOREM 13. Every infinite SI* group $G$ has an abelian subgroup $A \triangleleft^{2} G$ such that $\exp ^{2}|A| \geqq|G|$.

Theorem 6 sharpens Theorem 8 in [3]. Theorem 9 sharpens Corollary 1 in [2]. Theorem 13 sharpens Theorem 2 in [10]. Some of the results of this paper were previously announced in [5].

2. Notation. Let $S$ and $T$ be sets. $S<T$ always means strict inclusion. The cardinality of $S$ is denoted by $|S|$. If $m$ is an infinite cardinal, $\Omega(m)$ is the initial ordinal of cardinality $m ; m^{+}$is the first cardinal greater than $m$; $\exp ^{1} m=\exp m=2^{m}$; $\exp ^{n+1}=\exp \exp ^{n} m$; and $\Omega\left(\boldsymbol{\aleph}_{\beta}\right)=\omega_{\beta}$. The cofinality of an ordinal $\gamma$ (cardinal $m$ ) is the first cardinal $n$ such that $\gamma(m)$ is the sum of $n$ smaller ordinals (cardinals); we denote this by $n=c f(\gamma)(c f(m)) ; m$ is a regular cardinal if $c f(m)$ $=m$ and singular otherwise. A stationary subset of $\omega_{\alpha}$ is a subset which meets every closed unbounded subset.

If $G$ is a group and $H$ is a subgroup, we write $H \triangleleft^{n} G$ if there is an ascending normal series

$$
H=H_{0} \triangleleft H_{1} \triangleleft \cdots \triangleleft H_{n}=G
$$

from $H$ to $G$. If $H$ is characteristic in $G$ write $H \square G$. $C(H)=C_{G}(H)$ $=C(H \leqq G)$ denotes the centralizer of $H$ in $G$, while $N(H)=N_{G}(H)$ $=N(H \leqq G)$ denotes the normalizer of $H$ in $G$. The automorphism group of $G$ is denoted by $\operatorname{Aut}(G) . Z_{\alpha}(G)$ will denote the $\alpha^{\text {th }}$ member of

Received by the editors on February 25, 1975, and in revised form on April 26, 1976. 
the ascending central series. $G$ is a $\mathrm{ZA}$ group if it is the union of all the terms of its ascending central series. The class of a $Z A$ group is the first $\alpha$ such that $Z_{\alpha}(G)=G$. $G$ is nilpotent if it is a ZA group with finite class. If $x, y \in G$, the commutator of $x$ and $y$ is $[x, y]=x^{-1} y^{-1} x y$. We denote the derived (commutator) group of $G$ by $G^{1}$. Let $G^{n+1}=$ $\left(G^{n}\right)^{1}$. If $G$ is solvable, the length of $G$ is the first $n$ such that $G^{n}=E$, where $E=\{1\}$ denotes the identity subgroup.

Let $F_{1}(G)$ be the set of all elements in $G$ which have at most a finite number of conjugates in G. Following [7], the ascending FC series of $G$ is the series

$$
E=F_{0}(G) \square F_{1}(G) \square \cdots \square F_{\alpha}(G) \square \cdots
$$

where $F_{\alpha+1}(G) / F_{\alpha}(G)=F_{1}\left(G / F_{\alpha}(G)\right)$, and if $\beta$ is a limit ordinal, then $F_{\beta}=\bigcup_{\alpha<\beta} F_{\alpha}(G)$. If $F_{1}(G)=G, G$ is an $F C$ group. If $F_{\alpha}(G)=G$ for some $\alpha$, then $G$ is a $Z F C$ group; if $\alpha$ is an integer, $G$ is $F C$ nilpotent.

If $m$ is an infinite cardinal, let $M_{1}(G)$ be the set of all elements in $G$ which have at most $m$ conjugates in $G$. By analogy with the ascending $F C$ series, we define the ascending $m C$ series of $G$ to be the series

$$
E=M_{0}(G) \square M_{1}(G) \square \cdots \square M_{\alpha}(G) \square \cdots
$$

where $M_{\alpha+1}(G) / M_{\alpha}(G)=M_{1}\left(G / M_{\alpha}(G)\right)$, and if $\beta$ is a limit ordinal, then $M_{\beta}(G)=\bigcup_{\alpha<\beta} M_{\alpha}(G)$. If $M_{1}(G)=G, G$ is an $m C$ group; if $M_{\alpha}(G)=G$ for some $\alpha$, then $G$ is a $Z m C$ group.

We assume the terminology of $\$ 57$ and $\$ 63$ of [11] to denote various classes of generalized solvable and nilpotent groups. In addition, if $\chi$ is a class of groups, $\chi I^{*}$ is the class of all groups having an ascending invariant series with factors in $\chi ; \chi J^{*}$ is the class of all groups having an ascending subnormal series with factors in $X$; and $\chi N^{*}$ is the class of all groups having an ascending normal series with factors in $\chi$. Additional terminology and facts concerning infinite groups can be found in [14] and [15].

3. Large abelian subgroups.

LEMMA 1. Let $G$ be an infinite group and $H$ a subgroup. If $H \leqq N$ $<G$ and $|N||H|<|G|$, then $|C(H)|=|G|$.

Proof. If $N$ is finite, $|C(N)|=|G|:$ If $N$ is infinite, $[G: C(H)]=$ $[G: N(H)][N(H): C(H)] \leqq|C l(H)| \mid$ Aut $\left.H|\leqq| N\right|^{|H|}<|G|$.

THEOREM 1 . If $G$ is a group of uncountable regular cardinality having a (strictly increasing) normal series $\left(G_{\alpha}\right)$ with $\left|G_{\alpha}\right|<|G|$ and if $H$ is a subgroup of $G$ with $\left|G_{\alpha}\right||H|<|G|$ for all $\alpha$, then $|C(H)|=|G|$. In particular, every maximal abelian subgroup $A$ of $G$ satisfies $m^{|A|}$ $\geqq|G|$ for some $m<|G|$. 
Proof. Let $\theta=\Omega(|G|)$. Well-order $G=\left\{x_{\alpha} \mid \alpha<\theta\right\}$ so that (1) $x_{\varepsilon} \in G_{\epsilon+1}$ and (2) $x_{\rho} \in G_{\epsilon}$ implies that $x_{\sigma} \in G_{\epsilon}$ for all $\sigma \leqq \rho$. Since $|H|<|G|, H \leqq\left\{x_{\alpha} \mid \alpha<\gamma\right\}$ for some $\gamma<\theta$. For each $h \in H$ and $\epsilon$ such that $x_{\epsilon} \notin G_{y+1}$, let $\mu_{h}(\epsilon)$ be defined by $x_{\mu_{h}(\epsilon)}=\left[h, x_{\epsilon}\right]$. Let $\sigma$ be the first ordinal such that $x_{\epsilon} \in G_{\sigma+1}$. Since $\left\{x_{\alpha} \mid \alpha<\gamma\right\} \leqq G_{\sigma} \triangleleft$ $G_{\sigma+1},\left[h, x_{\epsilon}\right] \in G_{\sigma}$. If $\epsilon \leqq \mu_{h}(\epsilon)$, then $x_{\epsilon} \in G_{\sigma}$, a contradiction. It follows that $\mu_{h}(\epsilon)<\epsilon$. Let $S=\{\epsilon \mid \epsilon>\gamma$ and $\operatorname{cf}(\epsilon)>|H|\}$. Let

$$
\mu(\epsilon)=\sup _{h \in H} \mu_{h}(\epsilon)
$$

$\mu(\epsilon)<\epsilon$ for $\epsilon \in S$ since $\operatorname{cf}(\epsilon)>|H|$. Now $|H|^{+}<|G|$, otherwise $2^{|H|}=|H|^{|H|} \geqq|G|$, so $S \supseteq\left\{\alpha<\left.\theta|\operatorname{cf}(\alpha)=| H\right|^{+}\right.$and $\left.\alpha>\gamma\right\}$. If $C$ is any closed unbounded subset of $\theta$ and if $\alpha$ is the first member of $C$ such that $|\{\beta \in C \mid \gamma<\beta<\alpha\}|=|H|^{+}$, then $\alpha$ is the sum of the ordinals $\beta \in C$ with $\beta<\alpha$ since $C$ is closed and hence $\operatorname{cf}(\alpha)=|H|^{+}$ since $|H|^{+}$is regular. Thus $\alpha \in S$ and it follows that $S$ is a stationary subset of $\theta$. By $[13 ;$ p. 260$]$, there exists $T \leqq S$ such that $\mu$ is constant on $T$ and $|T|=|G|$. (In fact, by $[6$, p. 141], $T$ can be taken to be a stationary subset of $G$.) Hence there exists $\tau<\theta$ such that [ $\left.h, x_{\epsilon}\right] \in G_{\tau}$ for all $\epsilon \in T$ and $h \in H$. Define an equivalence relation $\sim$ on $T$ by $\rho \sim \sigma$ if and only if for all $h \in H,\left[h, x_{\rho}\right]=\left[h, x_{\sigma}\right]$. Since the number of equivalence classes is less than or equal to the number of subsets of $G_{\tau}$ of cardinality $|H|$, there are at most $\left|G_{\tau}\right||H|<|G|$ equivalence classes and one must have cardinality $|G|$, that is, there exists $U \leqq T$ with $|U|=|G|$ such that for all $\rho, \sigma \in U$ and for every $h \in H,\left[h, x_{\rho}\right]=\left[h, x_{\sigma}\right]$. Then $x_{\sigma} x_{\rho}^{-1} h x_{\rho} x_{\sigma}{ }^{-1}=h$ for all $\rho, \sigma \in U$ and $h \in H$, so $\left\langle x_{\sigma} x_{\rho}{ }^{-1} \mid \sigma, \rho \in U\right\rangle \leqq C(H)$. This shows that $|C(H)|=$ $|G|$. If $A$ is a maximal abelian subgroup with $|A|<|G|$ and $\left|G_{\alpha}\right| A|<| G \mid$ for all $\alpha$, then $|A|=|C(A)|=|G|$, a contradiction.

REMARK 1. R. Laver and the author have observed that if one assumes the generalized continuum hypothesis (G.C.H.), one gets the following stronger theorem.

THEOREM 1'. Let $G$ be an uncountable group with a (strictly increasing) normal series $\left(G_{\alpha}\right)$ with $\left|G_{\alpha}\right|<|G|$. If $H$ is any subgroup of $G$ with $|H|<\mathrm{cf}|G|$ and $\left|G_{\alpha}\right||H|<|G|$ for all $\alpha$, then $|C(H)|=|G|$. In particular, every maximal abelian subgroup $A$ of $G$ satisfies either (1) $|A| \geqq \operatorname{cf}|G|$ or $(2) m^{|A|} \geqq|G|$ for some $m<|G|$.

Proof. Suppose $G$ is a counterexample of smallest (singular) cardinality. Since $|H|<\mathrm{cf}|G|$, there is some $\alpha$ such that $H \leqq G_{\alpha},|H|<\left|G_{\alpha}\right|$ and $|C(H)|<\left|G_{\alpha}\right|$. There are two cases. If there exists a $\gamma \geqq \alpha$ such that $\left|G_{\gamma+1}\right| \geqq\left|G_{\gamma}\right|^{++}$, then by G.C.H. $\left.\left|G_{\gamma}\right|\right|^{|H|}<\left|G_{\gamma+1}\right|$. Thus Lemma 1 applies and $|C(H)| \geqq\left|G_{\gamma+1}\right| \geqq\left|G_{\alpha}\right|$, a contradiction. On the other hand, if no such $\gamma$ exists, there is a first ordinal $\mu$ such that $\left|G_{\mu}\right|=\left|G_{\alpha}\right|^{++}$. By 
G.C.H., if $\beta<\mu$, then $\left|G_{\beta}\right|^{|H|} \leqq\left(\left|G_{\alpha}\right|^{+}\right)^{|H|}=\left|G_{\alpha}\right|^{+}<\left|G_{\mu}\right|$, so Theorem 1 applies and yields $|C(H)| \geqq\left|G_{\mu}\right| \geqq\left|G_{\alpha}\right|$, a contradiction.

THEOREM 2. Let $G$ be a group with $|G|>m$. Suppose $G$ has a normal series $\left(G_{\alpha}\right)$ such that $\left[G_{\alpha+1}: G_{\alpha}\right] \leqq m$ forall $\alpha+1$. Then Ghas an abelian subgroup A such that $m^{|A|} \geqq|G|$.

Proof. Let $\mathcal{X}=\sup \{|A| \mid A$ abelian $\}$ and suppose $n=\sup \left\{\left(m^{|A|}|+| A\right.\right.$ abelian $\} \leqq|G|$. If there exists $A$ such that $|A|=\boldsymbol{N}$, then $n=(m \times)^{+}$. By Theorem $1, G_{\Omega_{(n)}}$ has an abelian subgroup $A$ such that $\left|G_{\Omega_{(n)}}\right| \leqq(m \times)^{|A|}$ $\leqq m^{\kappa}$, contradicting $\left|G_{n(n)}\right|=\left(m^{\kappa}\right)^{+}$. On the other hand, if $\sup _{\alpha} m_{\alpha}=$ $\aleph$, every maximal abelian subgroup $A$ of $G_{\Omega\left(\left(m^{m_{\alpha}}\right)^{+}\right)}$has $|A|>m_{\alpha}$ so we can find an abelian subgroup of power $\boldsymbol{\aleph}$.

Question 1. Suppose $G$ is a group with $|G|>m$. If $G$ has a normal series $\left(G_{\alpha} \mid \alpha<\lambda\right)$ with $\lambda$ a limit ordinal and $\left[G_{\alpha+1}: G_{\alpha}\right] \leqq m$, does every maximal abelian subgroup satisfy $m^{|A|} \geqq|G|$ ?

THEOREM 3. Every infinite $\mathrm{SN}^{*}$ group $G$ has an abelian subgroup $A$ such that $\exp |A| \geqq|G|$.

Proof. $G$ has a normal series with countable factors. If $G$ is countable, it has an infinite abelian subgroup by $[10 ; p$. 243]. If $G$ is uncountable, Theorem 2 yields the result.

Example 1. ([15, p. 454]) For every cardinal $\boldsymbol{\kappa}_{\alpha}$ there is a twostep solvable group $G_{\alpha}$ with $\left|G_{\alpha}\right|=\aleph_{\alpha}$ and having a finite maximal abelian subgroup. Let $H_{\epsilon}=\left\langle x_{\epsilon}\right\rangle \cdot\left\langle y_{\epsilon}\right\rangle$ with $x_{\epsilon}{ }^{2}=1=y_{\epsilon}{ }^{2}$. Then $H_{\alpha}=\sum_{\epsilon<\omega_{\alpha}} H_{\epsilon}$ has an automorphism $a_{\alpha}$ of order three given by $a_{\alpha}\left(x_{\epsilon}\right)=y_{\epsilon}$ and $a_{\alpha}\left(y_{\epsilon}\right)=x_{\epsilon} y_{\epsilon}$. If $G_{\alpha}$ is the split extension $\left\langle a_{\alpha}\right\rangle H_{\alpha},\left\langle a_{\alpha}\right\rangle$ is a maximal abelian subgroup.

THEOREM 4. [4, p. 31] If $G$ is an infinite FC group, every maximal abelian subgroup $A$ has $\exp |A| \geqq|G|$. If $G$ is an infinite $m C$ group, every maximal abelian subgroup $A$ has $m^{|A|} \geqq|G|$.

Proof. Since $A \leqq A^{G} \triangleleft G$, Lemma 1 applies. If $|A|<|G|$, then $|G| \leqq\left|A^{G}\right||A| \leqq|\langle\mathrm{Cl}(x) \mid x \in A\rangle||A|$. If $G$ is $F C, A$ cannot be finite since $G$ is infinite. Thus $|G| \leqq\left(|A| \cdot \boldsymbol{N}_{0}\right)^{|A|}=\exp |A|$. If $G$ is $m C,|G|$ $\leqq(m \cdot|A|)^{|A|}=m^{|A|}$.

THEOREM 5. Every $m C N^{*}$ group $G$ has a normal series $\left(G_{\alpha}\right)$ such that $\left[G_{\alpha+1}: G_{\alpha}\right] \leqq m$ for all $\alpha+1$.

Proof. Let $\left(G_{\alpha}\right)$ be any ascending $m C$ series for $G$. Let $G_{\alpha, 0}=G_{\alpha}$ for all $\alpha$. We form an invariant series $\left(G_{\alpha, \beta} / G_{\alpha}\right)$ for the $m C$ group $G_{\alpha+1} / G_{\alpha}$ by choosing $G_{\alpha, \beta+1} / G_{\alpha, \beta}$ to be any normal subgroup of $G_{\alpha+1} / G_{\alpha, \beta}$ of cardinality at most $m$. It follows that $\left(G_{\alpha, \beta}\right)$ is the desired normal series. 
THEOREM 6. Every infinite FCN* group $G$ has an abelian subgroup A such that $\exp |A| \geqq|G|$. Every $m C N^{*}$ group $G$ has an abelian subgroup A such that $m^{|A|} \geqq|G|$.

Proof. This theorem follows directly from Theorems 2 and 5 except in the case where $G$ is a countable $F C N^{*}$ group. In that case, we may suppose that $G$ has an $F C$ series $\left(G_{\alpha} \mid \alpha \leqq \beta\right)$ of minimal length. Then $G_{\alpha}$ is finite for all $\alpha<\beta$. If $\beta=\omega, G$ is locally finite and has an infinite abelian subgroup by $\left[15, \mathrm{p}\right.$. 453]. If $\beta=n+1, G / G_{n}$ is $F C$ and thus has an infinite abelian subgroup $H / G_{n}$ by Theorem 4 . Since $H^{1} \leqq G_{n}$ is finite, $H$ is $F C$ and the theorem follows from Theorem 4.

LEMMA 2. If $A$ is an abelian subgroup of $G$, then $A Z_{\lambda+1}(G)$ is at most a class $\lambda+1 \mathrm{ZA}$ group.

Proof. Note that $A Z_{\lambda+1}(G) / Z_{\lambda}(G)$ is abelian and that $Z_{\lambda}(G) \leqq$ $\mathrm{Z}_{\lambda}\left(A Z_{\lambda+1}(G)\right)$.

THEOREM 7. Let $A$ be a maximal abelian subgroup of an infinite nilpotent group $G$. Then

$$
\exp |A| \geqq|G| \text {. }
$$

Proof. We suppose $G$ is a counter-example of smallest class $\lambda$. Since $G^{1} \leqq Z_{\lambda-1}(G)$, if $Z_{\lambda-1}(G)$ is finite, $G$ is $F C$ and the result follows from Theorem 4. Thus $\exp |A| \geqq\left|A Z_{\lambda-1}\right| \geqq\left|Z_{\lambda-1}(G)\right| \geqq\left|G^{1}\right|$, so $G$ is $(\exp |A|) C$. Thus

$$
|G| \leqq(\exp |A|)^{|A|}=\exp |A| .
$$

Theonem 8. Let $G$ be an infinite $\mathrm{ZA}$ group with a finite maximal abelian subgroup $A$. Then $G$ is a direct sum of Černikov p-groups for a finite number of distinct primes $p$. In particular, $G$ is countable.

Proof. First we show that $G$ is periodic. Suppose $\alpha+1$ is the first ordinal such that there exists an element $x$ with infinite order in $Z_{\alpha+1}$. Then $Z_{\beta}$ is periodic for $\beta \leqq \alpha$. If $a \in A$, then $[x, a] \in Z_{\alpha}$. Suppose for all $n,\left[x^{n}, a\right] \neq 1$. Then let $\lambda+1$ be the first ordinal such that there exists $n,\left[x^{n}, a\right] \in Z_{\lambda+1}$. Then $\lambda<\alpha$, so $Z_{\lambda+1}$ is periodic and there exists $n$ such that $\left[x^{n}, a\right] \in Z_{\lambda+1}$. Thus $a^{-1} x^{n} a=x^{n} z, z \in$ $Z_{\lambda+1}$. Since $[z, x] \in Z_{\lambda}$, if $m$ is the order of $z,\left(a^{-1} x^{n} a\right)^{m} Z_{\lambda}=\left(x^{n} z\right)^{m} Z_{\lambda}$ $=x^{n m} z^{m} Z_{\lambda}=x^{n m} Z_{\lambda}$. Therefore $\left[x^{n m}, a\right] \in Z_{\lambda}$, a contradiction. This shows that for each $a \in A$ there exists $n(a)$ such that $x^{n(a)} \in C(a)$. Let $n=\prod_{a \in A} n(a) . \quad A$ cannot be finite since $\left\langle x^{n}\right\rangle \leqq C(A)=A$. This proves that $G$ is periodic.

Now suppose $G$ is a $p$-group. Let $A_{0}$ be a maximal normal abelian subgroup. $A_{0}$ is an infinite maximal abelian subgroup of $G$ by $[3, p$. 681]. Let $V=\left\{x \in A_{0} \mid x^{p}=1\right\}$. Suppose, by way of contradiction, 
that $V$ is infinite. Since $V$ is a characteristic subgroup of $A_{0}$, we may suppose $G=A V, V \triangleleft G$. Suppose $W$ is a finite subgroup of $V$ and $W^{A} \leqq W$. If $H / W=Z(G / W)$ is infinite, $A H$ is an infinite $F C$ group. By Theorem 4, A cannot be a maximal abelian subgroup of $A H$, a contradiction. Thus $Z(G / W)$ must be finite. We claim that $V$ has a minimal infinite subgroup $V_{0}$ such that $V_{0}{ }^{A} \leqq V_{0}$. Let $\left\{V_{i}\right\}_{i \in I}$ be a descending chain of infinite subgroups such that $V_{i}{ }^{A} \leqq V_{i}$. Suppose $W=\bigcap_{i \in I} V_{i}$ is finite. Then $Z(G / W) \neq E$ is finite. We also know that $V_{i} / W \cap Z(G / W) \neq E$ (see $\left[14\right.$, p. 14]). Thus $E \neq \bigcap_{i \in I}\left(V_{i} / W \cap\right.$ $Z(G / W))=W / W \cap Z(G / W)$, a contradiction. Thus $W$ is infinite, and the claim follows by Zorn's Lemma. Consider $\left(A V_{0}\right)^{i} \leqq V_{0}$. It is easy to calculate that $\left(A V_{0}\right)^{i} \leqq(A-1) V_{0} \equiv\left\langle v^{\alpha}-v \mid v \in V_{0}, \alpha \in A\right\rangle$. Since for every $\alpha \in A$ there exists $k$ such that

$$
(\alpha-1)^{p k}=\alpha^{p k}-1=0,
$$

where $\alpha-1$ is the endomorphism on $V$ defined by $(\alpha-1)(v)=$ $v^{\alpha}-v,(\alpha-1) V_{0}$ is a proper subgroup of $V_{0}$ such that $\left[(\alpha-1) V_{0}\right]^{A} \leqq$ $(\alpha-1) V_{0}$. Thus $(\alpha-1) V_{0}$ is finite and so is $(A-1) V_{0}$. It follows that $\left(A V_{0}\right)^{1}$ is finite. Hence $A V_{0}$ is an infinite $F C$ group, contradicting Theorem 4. This shows that $V$ is finite. Hence $A_{0}=D+R$ where $D$ is a finite sum of $p^{\infty}$ groups and $R$ is reduced. Since every infinite reduced group contains a cyclic summand, if $R$ is infinite it contains an infinite direct sum of cyclic groups, contradicting the fact that $V$ is finite. Thus $R$ is finite and $A_{0}$ satisfies Min. Since $A_{0}$ is maximal, $G / A_{0}$ is a periodic group of automorphisms of $A_{0}$. By a theorem of Baer (see [14, p. 54]), $G A_{0}$ satisfies Min. It follows that $G$ is a Černikov $p$-group.

Now since $G$ is periodic, $G$ is the direct sum of non-trivial $p$ groups for different primes $p, G=\sum G_{p}$. A is also the direct sum of p-groups, $A=\sum A_{p}$, where each $A_{p}$ must be a maximal abelian subgroup of $G_{p}$. Hence $G$ is a finite sum of Černikov $p$-groups, and $G$ is countable.

Example 2. Let $H$ be the direct sum of $Z_{3} \infty$ groups generated by $\left\{x_{i} \mid 3 x_{i+1}=x_{i}\right\}$ and $\left\{y_{i} \mid 3 y_{i+1}=y_{i}\right\}$. Let $\alpha$ be the automorphism of order 3 on $H$ defined by $\alpha\left(x_{i}\right)=-x_{i}+y_{i}$ and $\alpha\left(y_{i}\right)=-x_{i}$. The split extension $\langle\alpha\rangle H$ is a Cernikov 3-group. Suppose $z \in H$ and $\alpha(z)=z$. Then since $z=a x_{i}+b y_{i}$ for some $i$ with $a, b \in Z_{3}{ }^{i}$, we have $3 a x_{i}=3 b y_{i}=0$. Thus $z \in\left\langle x_{1}+y_{1}\right\rangle$ and so $\langle\alpha\rangle \cdot\left\langle x_{1}+y_{1}\right\rangle$ is a finite maximal abelian subgroup.

THEOREM 9. Let $A$ be a maximal abelian subgroup of an uncountable ZA group G. Then

$$
\exp |A| \geqq|G| \text {. }
$$


Proof. We suppose that $G$ is a counter-example of smallest class $\lambda+n$, where $\lambda$ is a limit ordinal. By hypothesis,

$$
\exp |A| \geqq\left|A Z_{\alpha+1}(G)\right| \geqq\left|Z_{\alpha+1}(G)\right|
$$

for all $\alpha+1<\lambda+n$ such that $Z_{\alpha+1}(G)$ is uncountable. Also, by Theorem 8, we may suppose that $|G|>\aleph_{1}$ and that $A$ is infinite. Hence $\exp |A| \geqq\left|Z_{\alpha+1}(G)\right|$ for all $\alpha+1<\lambda+n$. If $n=0, G=$ $\bigcup_{\alpha<\gamma} Z_{\alpha+1}(G)$. Thus $\exp |A| \geqq \lim _{\alpha<\lambda}\left|Z_{\alpha+1}(G)\right|$. If $n=1, \exp |A| \geqq$ $\lim _{\alpha<\lambda}\left|Z_{\alpha+1}(G)\right| \geqq\left|Z_{\lambda}(G)\right| \geqq\left|G^{1}\right|$. If $n>1, \exp |A| \geqq\left|Z_{\lambda+n-1}(G)\right| \geqq$ $\left|G^{1}\right|$. In either case, $G$ is $(\exp |A|) C$ and by Theorem 4 ,

$$
|G| \leqq(\exp |A|)^{|A|}=\exp |A| .
$$

ReMarx 2. The group $G_{\alpha}$ of Example 1 is a two-step $F C$ nilpotent group. Thus Theorem 9 cannot be extended to $F C$ nilpotent groups.

Question 2. Is there a nilpotent group $G$ with a maximum abelian subgroup $A$ such that exp $\mid$ core $A|<| G \mid$ ?

Question 3. Does every infinite $F C N^{*}$ group have an equipotent SN* subgroup?

THEOREM 10. Every infinite solvable group $G$ of length $n$ has a characteristic nilpotent subgroup $N$ of class at most $n$ such that $\exp |N| \geqq|G|$.

Proof. We induct on $n$. The theorem is clearly true for $n=1$. Let $n+1$ be the length of a counter-example $G$ of shortest length. We must have $\exp \left|G^{n}\right|<|G|$. Thus

$$
\left[G: C\left(G^{n}\right)\right] \leqq \mid \text { Aut } G^{n}|\leqq \exp | G^{n}|<| G \mid \text {. }
$$

Hence $H=C\left(G^{n}\right) \square G$ has the same cardinality as $G$. Since $G^{n} \subseteq$ $Z(H) \square H$, we must have $|Z(H)|<|H|$. Since $H / Z(H)$ has shorter solvable length, it has a characteristic nilpotent subgroup $N / Z(H)$ of class at most $n$ such that $\exp |N| Z(H)|\geqq| H / Z(H)|=| H \mid$. Thus $[N, N, \cdots, N] \leqq Z(H)$. Since $[N, Z(H)]=E, N$ is a nilpotent group of class at most $n+1$. In addition, since $N / Z(H) \square H / Z(H)$ and $Z(H) \square H$, $N \square H \square G$ and

$$
\exp |N| \geqq \exp |N / Z(H)| \geqq|H|=|G| .
$$

THEOREM 11. An infinite solvable group $G$ of length $n$ has a characteristic two-step nilpotent subgroup $N$ such that $\exp ^{n}|N| \geqq|G|$.

Proof. As above, let $n+1$ be the length of a counter-example $G$ of shortest length. If $\exp \left|G^{1}\right|<|G|$, we have 


$$
\left[G: C\left(G^{1}\right)\right] \leqq\left|\operatorname{Aut}\left(G^{1}\right)\right| \leqq \exp \left|G^{1}\right|<|G|,
$$

so $\left|C\left(G^{1}\right)\right|=|G|$. Since $C\left(G^{1}\right)$ is a characteristic two-step nilpotent subgroup, we must have $\exp \left|G^{1}\right| \geqq|G|$. Since $G^{1}$ has a characteristic two-step nilpotent subgroup $N$ such that $\exp ^{n}|N| \geqq\left|G^{1}\right|$, we have $\exp ^{n+1}|N| \geqq \exp \left|G^{1}\right| \geqq|G|$.

Remark 3. There is an infinite two-step solvable group all of whose normal abelian subgroups are finite. See [8].

LeMMA 3. [14, p. 14]. Let $E \neq H \triangleleft G$. If $G$ is $S I^{*}, H$ contains a non-trivial abelian subgroup normal in $G$. If $G$ is $F C I^{*}, H$ contains a non-trivial FC subgroup normal in $G$.

Proof. We shall prove only the second statement. Let $\left(G_{\alpha}\right)$ be an invariant $F C$ series for $G$. Let $\alpha$ be the least ordinal such that $H \cap G_{\alpha}$ $\neq E$. Since $\alpha$ is not a limit ordinal, $H \cap G_{\alpha-1}=E$ and $H \cap G_{\alpha} \cong(H$ $\left.\cap G_{\alpha}\right) G_{\alpha-1} / G_{\alpha-1} \leqq G_{\alpha} / G_{\alpha-1}$. Thus $H \cap G_{\alpha} \triangleleft G$ is the desired group.

THEOREM 12. Every infinite SI* group $G$ has a normal ZA subgroup $H$ such that $\exp |H| \geqq|G|$. Every infinite $F C I^{*}$ group $G$ has a normal ZFC subgroup $H$ such that $\exp |H| \geqq|G|$.

Proof. We shall prove only the second statement. Let $H_{0}=G$ and let $A_{0}=E$. If possible, let $A_{\alpha+1}$ be a normal subgroup of $G$ such that $A_{\alpha+1} / A_{\alpha}$ is a non-trivial normal $F C$ subgroup of $G / A_{\alpha}$ contained in $H_{\alpha} / A_{\alpha}$. Then let $H_{\alpha+1}$ be the normal subgroup of $G$ such that $H_{\alpha+1} / A_{\alpha}$ $=\left(A_{\alpha+1} / A_{\alpha}\right) C\left(A_{\alpha+1} / A_{\alpha} \leqq H_{\alpha} / A_{\alpha}\right)=\left(A_{\alpha+1} / A_{\alpha}\right)\left(C\left(A_{\alpha+1} / A_{\alpha}\right) \cap H_{\alpha} / A_{\alpha}\right)$. If $\beta$ is a limit ordinal, let $A_{\beta}=\bigcup_{\alpha<\beta} A_{\alpha}$ and let $H_{\beta}=\bigcap_{\alpha<\beta} H_{\alpha}$. Since $G / A_{\alpha}$ is an $\mathrm{FCI}^{*}$ group, if $H_{\alpha} / \mathrm{A}_{\alpha}$ is not trivial, then it contains a non-trivial $F C$ subgroup normal in $G / A_{\alpha}$ by Lemma 3 . Hence $A_{\alpha+1}>A_{\alpha}$ unless $H_{\alpha}=$ $A_{\alpha}$. Thus there is a first ordinal $\gamma$ such that $H_{\gamma}=A_{\gamma}$.

Since $A_{\alpha+1} / A_{\alpha}$ is an $F C$ group, using the definition of $H_{\alpha+1}$, we see that each element in $A_{\alpha+1} / A_{\alpha}$ has only a finite number of conjugates in $A_{\gamma} / A_{\alpha}$. Thus $A_{\gamma}$ is a $Z F C$ group. We have (see [3, Theorem 1])

$$
\begin{aligned}
{\left[G: H_{\gamma}\right] } & \leqq \prod_{\alpha<\gamma}\left[H_{\alpha}: H_{\alpha+1}\right]=\prod_{\alpha<\gamma}\left[H_{\alpha} / A_{\alpha}: H_{\alpha+1} / A_{\alpha}\right] \\
& =\prod_{\alpha<\gamma}\left[N\left(A_{\alpha+1} / A_{\alpha} \leqq H_{\alpha} / A_{\alpha}\right):\left(A_{\alpha+1} / A_{\alpha}\right) C\left(A_{\alpha+1} / A_{\alpha} \leqq H_{\alpha} / A_{\alpha}\right)\right] \\
& \leqq \prod_{\alpha<\gamma}\left[N\left(A_{\alpha+1} / A_{\alpha} \leqq H_{\alpha} / A_{\alpha}\right): C\left(A_{\alpha+1} / A_{\alpha} \leqq H_{\alpha} / A_{\alpha}\right)\right] \\
& \leqq \prod_{\alpha<\gamma} \operatorname{Aut}\left(A_{\alpha+1} / A_{\alpha}\right) .
\end{aligned}
$$


It follows (see $[3, \S 3])$ that $\left[G: H_{\gamma}\right] \leqq \prod_{\alpha<\gamma} \boldsymbol{\kappa}_{0}\left|A_{\alpha+1} / A_{\alpha}\right| \leqq$ $\boldsymbol{N}_{.0}{ }^{\Sigma_{\alpha<\gamma}\left|A_{\alpha+1} / A_{a}\right|} \leqq \boldsymbol{N}_{0}{ }^{\left|A_{\gamma}\right|}=\exp \left|A_{\gamma}\right|$. Thus $|G| \leqq\left|H_{\gamma}\right| \exp \left|H_{\gamma}\right|$. Therefore $|G| \leqq \exp \left|H_{y}\right|$.

THEOREM 13. Every infinite $S I^{*}$ group $G$ has an abelian subgroup $A \triangleleft^{2} G$ such that $\exp ^{2}|A| \geqq|G|$.

Proof. This follows from Theorem 12 and the fact $[3$, p. 681] that every maximal normal abelian subgroup $A$ of a $Z A$ group $H$ satisfies $\exp |A| \geqq|H|$.

Remark 4. (See also Remark 3.) There is a non-abelian SI* group which has no nontrivial characteristic subgroups. (See [12] and $[14$, p. 102].) There is an SJ* group which has no non-trivial normal abelian subgroups. (See [1]).

Question 4. Can Theorem 13 be generalized to $\mathrm{FCI}^{*}$ groups?

Question 5. Can Theorem 13 be improved to read $\exp |A| \geqq|G|$ ?

THEOREM 14. Every infinite $m C I^{*}$ group $G$ has a normal $\mathrm{ZmC}$ subgroup $H$ such that $\exp |H| \geqq|G|$.

Proof. The proof is similar to that of Theorem 12; details are left to the reader.

REMark 5. M. J. Tomkinson [16] informs us that the construction used in $\$ 4$ of [9] can be used to construct without the continuum hypothesis a two-step nilpotent group of cardinality $2^{\mathrm{N}_{0}}$ all of whose maximal abelian subgroups have cardinality $\boldsymbol{\kappa}_{0}$. This construction does not seem to generalize to higher cardinalities and (unlike [2] where the continuum hypothesis is used) does not yield FC groups.

4. Acknowledgement. The author wishes to express his gratitude to M. Magidor and R. McKenzie who provided many helpful ideas which were incorporated in this work. Part of the work of the author was performed under a Summer Research Initiation Faculty Fellowship from the University of Colorado.

\section{REFERENCES}

1. R. S. Dark, A prime Baer group, Math. Zeit. 105 (1968), 294-298.

2. A. Ehrenfeucht and V. Faber, Do infinite nilpotent groups always have equipotent abelian subgroups? Indag. Math. 34 (1972), 202-209.

3. V. Faber, Large abelian subgroups of some infinite groups, Rocky Mountain Journal of Mathematics 1 (1971), 677-685.

4. - Chain index theorems and their applications in infinite groups, Ph.D. Thesis, Wash. Univ., St. Louis (1971). 
5. - Large abelian subgroups of infinite solvable and nilpotent groups, AMS Notices 19 (1972), A-386.

6. G. Fodor, Eine Bemerkung zur Theorie der regessiven Funktionen, Acta Sci. Math. (Szeged) 17 (1956), 139-142.

7. F. Haimo, The FC-chain of a group, Canad. J. Math. 5 (1953), 498-511.

8. H. Heineken and I. J. Mohamed, A group with trivial centre satisfying the normalizer condition, Journal of Alg. 10 (1968), 368-376.

9. L. G. Kovács, B. H. Neumann and H. de Vries, Proc. Royal Soc. of London $\Lambda 260$ (1961), 304-316.

10. C. R. Kulatilaka, Infinite abelian subgroups of some infinite groups, J. London Math. Soc. 39 (1964), 240-244.

11. A. G. Kuroš, The Theory of Groups, vol. II, translated and edited by K. A. Hirsch, Chelsea Publishing Co., New York (1960).

12. D. H. McLain, A characteristically-simple group, Proc. Cambridge Philos. Soc. 50 (1954), 641-642.

13. W. Neumer, Verallgemeinerung eines Satzes von Alexandroff und Urysohn, Math. Zeit. 54 (1951), 254-261.

14. D. J. S. Robinson, Infinite Soluble and Nilpotent Groups, Queen Mary College Mathematics Notes, London (1967).

15. W. R. Scott, Group Theory, Prentice Hall, Englewood Cliffs (1964).

16. M. J. Tomkinson, private communication.

University of Colorado, Denver, Colorado 80202 